\title{
A Survey on Safe Information Sharing and Query Processing Via Organization of Cloud Computing
}

\author{
Shraddha Jadhav \\ ME Student, Dept. of Computer Engineering, Sinhgad Academy of Engg. Pune, Maharashtra, India
}

\begin{abstract}
Here, we are going to examine security issues for distributed computing and present a layered structure for secure mists and after that attention on two of the layers, i.e., the capacity layer and the information layer. Distributed computing is an arrangement of IT administrations that are given to a client over a system on a rented premise and with the capacity to scale up or down their administration necessities. Typically distributed computing administrations are conveyed by an outsider supplier who claims the base. It points of interest to specify yet a couple incorporate adaptability, strength, adaptability, proficiency and outsourcing non-center exercises. This paper will chat secure unified inquiry handling with guide Reduce and Hadoop, and examine the utilization of secure coprocessors for distributed computing. Distributed computing offers a creative plan of action for associations to receive IT administrations without forthright venture. Regardless of the potential additions accomplished from the distributed computing, the associations are moderate in tolerating it because of security issues and difficulties connected with it. Security is one of the real issues which hamper the development of cloud. The thought of giving over essential information to another organization is troubling; such that the buyers need to be careful in comprehension the dangers of information ruptures in this new environment. This paper presents a nitty gritty examination of the distributed computing security issues and difficulties concentrating on the distributed computing sorts and the administration conveyance sorts.
\end{abstract}

Keywords: Cloud computing, Security, Data Storage, Data Encryption, Data Decryption.

\section{Introduction}

There is a basic need to safely store, oversee, offer and break down gigantic measures of complex (e.g., semi-organized what's more, unstructured) information to decide examples and patterns in request to enhance the nature of human services, better protect the country and investigate elective vitality. In light of the basic nature of the applications, it is imperative that mists be secure. The real security challenge with mists is that the proprietor of the information might not have control of where the information is set. This is on account of if one needs to abuse the advantages of utilizing cloud figuring, one must likewise use the asset designation and booking gave by mists. Consequently, we have to shield the information amidst untrusted forms. The developing distributed computing model endeavors to address the unstable development of web-joined gadgets, and handle monstrous measures of information. Google has now presented the MapReduce system for preparing a lot of information on item equipment. Apache ${ }^{\text {ee }}$ Hadoop disseminated record framework (HDFS) is developing as an unrivaled programming segment for distributed computing joined with coordinated parts, for example, MapReduce. The need to enlarge human thinking, translating, and choice making capacities has brought about the development of the Semantic Web, which is an activity that endeavors to change the web from its current, only human readable structure, to a machine-processable structure. This thus has brought about various informal communication destinations with enormous measures of information to be shared and oversaw. Subsequently, we desperately require a framework that can scale to handle a vast number of locales and procedure enormous measures of information. Notwithstanding, state of the workmanship frameworks using HDFS and MapReduce are most certainly not adequate because of the way that they don't give satisfactory security instruments to ensure touchy information. We are directing examination on secure distributed computing. Because of the broad intricacy of the cloud, we battle that it will be hard to give a comprehensive answer for securing the cloud, at present. Hence, we will likely make increase upgrades to securing the cloud that will at last result in a protected cloud. Specifically, we are adding to a protected cloud comprising of equipment (incorporates 800TB of information stockpiling on a mechanical plate drive, 2400 GB of memory and a few merchandise PCs), programming (incorporates Hadoop) and information (incorporates a semantic web information store). Our cloud framework will:

a) Support effective capacity of scrambled delicate information,

b)Store, oversee and inquiry gigantic measures of information,

c) Support fine-grained access control and Support solid confirmation.

This paper portrays our way to deal with securing the cloud.

\section{Literature Review}

\subsection{Cloud Computing}

Cloud computing is a web based processing where clients can share their information, assets, programming and it can likewise give data to PCs and numerous different gadgets on interest. Distributed computing is another innovation that conveys numerous sorts of assets on the web. In this way, distributed computing uses web as a correspondence medium to convey its administrations. Distributed computing can be offered inside of endeavors through LANs however as a general rule, it doesn't work all inclusive without web. Numerous endeavors and different associations need to store and work on a colossal measure of information. Distributed 


\section{International Journal of Science and Research (IJSR) \\ ISSN (Online): 2319-7064}

Index Copernicus Value (2013): 6.14 | Impact Factor (2014): 5.611

computing goes for leasing such assets on interest [10]. The National Institute of Standards and Technology (NIST) characterizes distributed computing as a model for helpful, on-interest system access to processing assets, for example, systems, servers, stockpiling, applications and so forth. "Cloud" alludes to the Web, mists can be open, private, or half and half (a mix of both open and private clouds)[4].

\subsection{Cloud Deployment Module}

a) Public Cloud: out in the open cloud, the cloud framework is accessible to the overall population.

b)Private Cloud: In private cloud, the cloud is accessible exclusively for a solitary association.

c) Community Cloud: In this sort of cloud arrangement demonstrate, the base of the cloud is shared by a few associations and backings a particular group with shared concerns [6].

\subsection{Architectural Layers of Cloud Computing}

1) Software as an administration (SaaS) Software as a Service elements a complete application offered as an administration on interest. A solitary occurrence of the product keeps running on the cloud and administrations various end clients or customer associations.

2)Platform as an administration (PaaS) This administration exemplifies a layer of programming and gives it as an administration that can be utilized to assemble more elevated amount administrations.

3)Infrastructure as an administration (IaaS) Infrastructure as an administration conveys fundamental stockpiling and figure capacities as institutionalized administrations over the system [9].

\subsection{Cloud Computing Evolution}

There has dependably been a verbal confrontation about the advancement of Cloud Computing and Grid Computing. A few individuals imagine that both chip away at same wonders while others call Cloud Computing as an augmentation of Grid processing. The table underneath demonstrates that Distributed computing is not the same as Grid registering. Distributed computing utilize the idea of virtualization to amplify the processing force. Virtualization, by isolating the coherent from the physical, determines a portion of the difficulties confronted by lattice processing" .

\subsection{Various Attacks In Cloud Environment}

Cloud Computing is the beginning stage and premise for Cloud Computing. Distributed computing essentially speaks to the expanding pattern towards the outer organization of IT assets, for example, computational force, stockpiling or business applications, and acquiring them as administrations [6]. Distributed computing is a quickly developing zone in figuring exploration and industry today. It has the potential to make the not all that new thought of 'figuring as an utility' a reality in the close future.[8]Cloud computing is getting to be a standout amongst the most tempting innovations, in light of its expense effectiveness and adaptability. Different security issues in the cloud are obstructing the vision of distributed computing as another IT acquirement model. Existing cryptographic strategies can be used for information security, however security assurance and outsourced calculation need critical consideration - both are generally new research headings [8]. Huge numbers of the organizations encode your records while they are in transit to the cloud and protect your records while away. Distributed computing gives access to information, yet the test is to guarantee that just approved elements can access it[2]. Information as a Service (DaaS) is developing as an essential administration model in which information put away in the cloud is made accessible to clients in light of their entrance criteria (chose by the information owner). On one hand, the lawful ramifications of the information (particularly in distributed computing information area matters as the laws representing the information vary over geographic limits) and applications being held by an outsider are mind boggling and are not surely knew [1]. Our examination center is to give an answer for the dangers that are the significant issue for anybody when they need to receive cloud administrations for their work. For this reason, a structure ought to be intended for execution of information and data safely in cloud environment. It will secure clients' information, messages, data against different assault.

\section{Security Issues in Cloud Computing}

\subsection{Cloud Deployments Models}

In the cloud sending model, organizing, stage, capacity, and programming base are given as administrations that scale up or down contingent upon the interest as delineated. The Cloud Computing model has three fundamental sending models which are:

\subsubsection{Private cloud}

Private cloud is another term that a few sellers have as of late used to depict offerings that imitate cloud processing on private systems. It is set up inside of an organization es inside big business datacenter. In the private cloud, versatile assets and virtual applications gave by the cloud merchant are pooled together and accessible for cloud clients to share and utilize. It contrasts from people in general cloud in that all the cloud assets and applications are overseen by the association itself, like Intranet usefulness. Usage on the private cloud can be substantially more secure than that of the open cloud as a result of its predetermined inside presentation. Just the association and assigned partners may have admittance to work on a particular Private cloud.[12]

\subsubsection{Public cloud}

Open cloud portrays distributed computing in the conventional standard sense, whereby assets are powerfully provisioned on a fine-grained, self-administration premise over the Web, by means of web applications/web administrations, from an off-website outsider supplier who offers assets and bills on a finegrained utility registering premise. It is commonly taking into account a payper-use model, like a prepaid power metering framework which is sufficiently adaptable to cook for spikes sought after for cloud optimization.[13] Public mists are less secure than the

\section{Volume 4 Issue 12, December 2015}




\section{International Journal of Science and Research (IJSR) \\ ISSN (Online): 2319-7064}

Index Copernicus Value (2013): 6.14 | Impact Factor (2014): 5.611

other cloud models in light of the fact that it puts an extra weight of guaranteeing all applications and information got to on people in general cloud are not subjected to noxious assaults

\subsubsection{Hybrid cloud}

Half breed cloud is a private cloud connected to one or more outer cloud administrations, halfway oversaw, provisioned as a single unit, and encompassed by a protected system [11]. It gives virtual IT arrangements through a blend of both open and private mists. Cross breed Cloud gives more secure control of the information and applications and permits different gatherings to get to data over the Internet. It likewise has an open building design that permits interfaces with other administration frameworks. Cross breed cloud can depict arrangement joining a nearby gadget, for example, a Plug PC with cloud administrations. It can likewise depict arrangements joining virtual and physical, assembled resources - for instance, a for the most part virtualized environment that requires physical servers, switches, or other equipment, for example, a system apparatus going about as a firewall or spam channe.

\subsection{Cloud Computing Service Delivery Models}

Taking after on the cloud arrangement models, the following security thought identifies with the different distributed computing administration conveyance models. The three primary cloud administration conveyance models are: Infrastructure-as-a-Service (IaaS),Stage as-a-Service (PaaS) and Software-as-a-Service (SaaS).

\subsubsection{Infrastructure as a Service (IaaS)}

Framework as a Service is a solitary occupant cloud layer where the Cloud registering vendores committed assets are just imparted to contracted customers at a pay-per-use charge. This incredibly minimizes the requirement for colossal beginning interest in processing equipment, for example, servers, organizing gadgets and preparing force. They additionally permit fluctuating degrees of budgetary and utilitarian adaptability not found in inner information focuses or with collocation administrations, in light of the fact that processing assets can be included or discharged a great deal all the more rapidly and expense viably than in an inside server farm or with a collocation administration [3]. IaaS and other related administrations have empowered new companies and different organizations concentrate on their center abilities without agonizing much over the provisioning what's more, administration of base. IaaS totally dreamy the equipment underneath it and permitted clients to expend foundation as an administration without trying anything about the basic complexities. The cloud has a convincing quality recommendation as far as expense, yet ,out of the box ${ }^{\text {ee }}$ IaaS just gives fundamental security (edge firewall, load adjusting, and so forth.) and applications moving into the cloud will need more elevated amounts of security gave at the host.

\subsubsection{Stage as an administration (PaaS)}

Stage as-a-Service (PaaS) is an arrangement of programming and improvement devices facilitated on the supplier's servers. It is one layer above IaaS on the stack and digests away everything up to OS, middleware, and so forth. This offers a coordinated arrangement of engineer environment that a designer can tap to fabricate their applications without having any piece of information about what is going on underneath the administration. It offers engineers an administration that gives a complete programming improvement life cycle administration, from wanting to outline to building applications to sending to testing to upkeep. Everything else is dreamy far from the "perspective" of the engineers. Stage as an administration cloud layer works like IaaS however it gives an extra level of ,rented ${ }^{\text {ee }}$ usefulness. Customers utilizing PaaS administrations exchange significantly more expenses from capital venture to operational costs however must recognize the extra imperatives and potentially some level of lock-in postured by the extra usefulness layers [11]. The utilization of virtual machines go about as an impetus in the PaaS layer in Cloud figuring. Virtual machines must be ensured against noxious assaults, for example, cloud malware. In this manner keeping up the uprightness of uses and well authorizing precise verification checks amid the exchange of information over the whole systems administration channels is basic.

\subsubsection{Software as a Service}

Programming as-a-Service is a product appropriation model in which applications are facilitated by a seller or administration supplier also, made accessible to clients over a system, commonly the Web. SaaS is turning into an undeniably predominant conveyance model as fundamental innovations that bolster web administrations furthermore, administration situated structural planning (SOA) experienced and new formative methodologies get to be mainstream. SaaS is additionally frequently connected with a pay-as-you-go membership permitting model. In the interim, broadband administration has turned out to be progressively accessible to bolster client access from more zones around the world. SaaS is frequently actualized to give business programming usefulness to big business clients requiring little to no effort while permitting those clients to acquire the same advantages of industrially authorized, inside worked programming without the related many-sided quality of establishment, administration, bolster, authorizing, and high starting expense. The structural planning of SaaS-based applications is particularly intended to bolster numerous simultaneous clients (multitenancy) immediately. Programming as a administration applications are gotten to utilizing web programs over the Web in this way web program security is crucially imperative.Data security officers should consider different strategies for securing SaaS applications. Web Services (WS) security, Extendable Markup Language (XML) encryption, Secure Socket Layer (SSL) and accessible alternatives which are utilized as a part of upholding information security transmitted over theInternet.[8] Consolidating the three sorts of mists with the conveyance models we get an all encompassing cloud outline .encompassed by network gadgets combined with data security subjects. Virtualized physical assets, virtualized foundation, and additionally virtualized middleware stages and business applications are being given and devoured as administrations in the Cloud [5]. Cloud sellers and clients need to keep up Cloud registering security at all interfaces. The following segment of the paper presents difficulties confronted in the cloud register.

\section{Volume 4 Issue 12, December 2015}




\section{Cloud Computing Challenges}

The present reception of distributed computing is connected with various difficulties in light of the fact that clients are still distrustful about its credibility. In view of a review led by IDC in 2008, the real difficulties that keep Cloud Computing from being received are perceived by associations are as per the following:

A. Security: It is clear that the security issue has played the most vital part in blocking Cloud figuring acknowledgment. Without uncertainty, putting your information, running your product on another person's hard circle utilizing another person's CPU shows up overwhelming to numerous. Understood security issues, for example, information mis fortune,phishing,botnet (running remotely on a gathering of machines) posture genuine dangers to association's information and programming. Additionally, the multi-tenure model and the pooled registering assets in distributed computing has presented new security challenges that require novel methods to handle with. For instance, programmers can utilize Cloud to arrange bot net as Cloud regularly gives more dependable foundation administrations at a moderately less expensive cost for them to begin an attack.[9]

B. Costing Model: Cloud shoppers must consider the tradeoffs amongst calculation, correspondence, and joining. While relocating to the Cloud can fundamentally lessen the base expense, it raises the expense of information correspondence, i.e. the expense of exchanging an association's information to and from general society and group Cloud and the expense per unit of figuring asset utilized is liable to be higher. This issue is especially unmistakable if the shopper employments the cross breed cloud sending model where the association's information is circulated amongst various open/private (inhouse IT framework)/group mists. Naturally, ondemand registering bodes well just for CPU concentrated jobs.[9]

C. Charging Model: The versatile asset pool has made the taken a toll investigation significantly more convoluted than general server farms, which frequently computes their expense taking into account utilizations of static figuring. In addition, an instantiated virtual machine has turned into the unit of expense investigation instead of the hidden physical server. For SaaS cloud suppliers, the expense of creating multitenancy inside of their offering can be extremely considerable. These include: re-configuration and redevelopment of the programming that was initially utilized for single-tenure, expense of giving new components that permit to escalated customization, execution and security upgrade for simultaneous client get to, and managing complexities prompted by the above changes. Therefore, SaaS suppliers need to weigh up the exchange off between the procurement of multitenancy and the costsavings yielded by multi-tenure, for example, diminished overhead through amortization, lessened number of on location programming licenses, and so forth. In this manner, a vital and suitable charging model for SaaS supplier is critical for the benefit and maintainability of SaaS cloud providers.[7]

\section{Secure Query Processing with Hadoop}

\section{Overview of Hadoop}

A noteworthy piece of our framework is HDFS which is a circulated Java-based document framework with the ability to handle an extensive number of hubs putting away peta bytes of information. In a perfect world a document size is a numerous of $64 \mathrm{MB}$. Dependability is accomplished by repeating the information over a few hosts. The default replication worth is 3 (i.e., information is put away on three hubs). Two of these hubs dwell on the same rack while the other is on an alternate rack. A bunch of information hubs builds the document framework. The hubs transmit information over HTTP and clientse access information utilizing a web program. Information hubs correspond with one another to manage, exchange and repeat information.

The expert is known as a Name node and contains metadata. It keeps the registry tree of all records and tracks which information is accessible from which hub over the bunch. This data is put away as a picture in memory. Information squares are put away in Data nodes. The name node is the single purpose of disappointment as it contains the metadata. In this way, there is discretionary auxiliary Name node that can be setup on any machine. The customer gets to the Name node to get the metadata of the required document. Subsequent to getting the metadata, the customer specifically converses with the particular Data nodes keeping in mind the end goal to get information or to perform IO activities (Hadoop). On top of the document frameworks there exists the guide/diminish motor. This motor comprises of a Job Tracker. The customer applications submit guide/diminish employments to this motor. The Work Tracker endeavors to put the work close to the information by pushing the work out to the accessible Task Tracker hubs in the bunch. Insufficiencies of Hadoop Current frameworks using Hadoop have the accompanying restrictions:

1)No office to handle encoded touchy information: Sensitive information running from therapeutic records to charge card exchanges should be put away utilizing encryption strategies for extra assurance. Presently, HDFS does not perform secure and proficient inquiry handling over encoded information

2)Semantic Web Data Management: There is a requirement for feasible answers for enhance the execution and adaptability of questions against semantic web information, for example, RDF (Resource Depiction Framework). The quantity of RDF datasets is expanding. The issue of putting away billions of RDF triples and the capacity to proficiently inquiry them is yet to be comprehended.

3)No fine-grained access control: HDFS does not give finegrained access control. There is some work to give access control records for HDFS.

4)No solid verification: A client who can unite with the JobTracker can present any employment with the benefits of the record used to set up the HDFS. Future renditions of HDFS will bolster system confirmation conventions like Kerberos for client verification and encryption of information exchanges. Be that as it may, for some 


\section{International Journal of Science and Research (IJSR) \\ ISSN (Online): 2319-7064}

Index Copernicus Value (2013): 6.14 | Impact Factor (2014): 5.611

guaranteed data sharing situations, we will require open key frameworks (PKI) to give computerized mark support.

\section{Conclusion}

In this paper, we initially talked about security issues for cloud. These issues incorporate capacity security, middleware security, information security, system security and application security. The fundamental objective is to safely store and oversee information that is most certainly not controlled by the operator of the information. Specifically, we are taking a base up way to deal with security where we are working on little issues in the cloud that we trust will explain the bigger issue of cloud security. We talked about how secure co-processors may be utilized to upgrade security. We executed the Hadoop at long last. There are some new advancements developing at a fast rate, each with mechanical headways and with the capability of making human ${ }^{\text {ee }}$ lives simpler. Be that as it may, one must be extremely cautious to comprehend the security dangers and difficulties postured in using these innovations. Distributed computing is no exemption. In this paper key security contemplations and difficulties which are as of now confronted in the Cloud registering are highlighted. Cloud registering can possibly turn into a leader in advancing a protected, virtual and monetarily reasonable IT arrangement later.

\section{References}

[1] Bharath K.Samanthula,Yousef Elmehdwi, Gerry Howser, Sanjay Madrian,"A secure data sharing and query processing framework via federation of cloud computing", Department of Computer Science, Missouri University of Science and Technology, 500 West 15th Street, Rolla, MO65401, United States, 2013

[2] Mark D. Ryan, "Cloud computing security: The scientific challenge, and a survey of solutions", The Journal of Systems and Software 86 (2013) 22632268.

[3] J. Brodkin. (2008, Jun.). Gartner: Seven cloudcomputing security risks." Infoworld, Available: [Mar. 13, 2009].

[4] Vairagade1, Nitin Ashokrao Vairagade 2,"Cloud Computing Data Storage and Security Enhancement", International Journal of Advanced Research in Computer Engineering \& Technology (IJARCET) Volume 1, Issue 6, August 2012.

[5] M. Klems, A. Lenk, J. Nimis, T. Sandholm and S. Tai. What ${ }^{\text {es }}$ Inside the Cloud? An Architectural Map of the Cloud Landscape.” IEEE Xplore, pp 23-31, Jun. 2009.

[6] Rehan Saleem (831015-T132),"-ELOUD COMPUTING'S EFFECT ON ENTERPRISES" - . . in terms of Cost and Security", January, 2011.

[7] S. Ramgovind, M. M. Eloff, E. Smith. The Management of Security in Cloud Computing" In PROC 2010 IEEE International Conference on Cloud Computing 2010.

[8] Bhathiya Wickremasinghe,"CloudAnalyst:A CloudSimbased Tool for Modelling and Analysis of Large Scale Cloud Computing EnvironmentsT,"433- 659
DISTRIBUTED COMPUTING PROJECT, CSSE DEPT., UNIVERSITY OF MELBOURNE,2009.

[9] Sun microsystem,"'Introduction to Cloud Computing architecture", White Paper 1st Edition, June 200910.

[10] Sven Bugiel1, Stefan Nurnberger1,Ahmad-Reza Sadeghi1,Thomas Schneider2,'TwinClouds:An Architecture for Secure Cloud Computing", Center for Advanced Security Research Darmstadt, Technische University at Darmstadt, Germany.

[11] Global Netoptex Incorporated. Đemystifying the cloud. Important opportunities, crucial choices." pp4-14. Available: http://www.gni.com [Dec. 13, 2009].

[12] S. Arnold (2009, Jul.). - Cloud computing and the issue of privacy." KM World, pp14-22. Available: www.kmworld.com [Aug. 19, 2009].

[13] A Platform Computing Whitepaper. Enterprise Cloud Computing: Transforming IT." Platform Computing, pp6, 2010. 